\title{
Bioabsorbable self-retaining PLA/nano-sized $\beta$-TCP cervical spine interbody fusion cage in goat models: an in vivo study
}

This article was published in the following Dove Press journal:

International Journal of Nanomedicine

3 October 2017

Number of times this article has been viewed

\author{
Lu Caol,* \\ Qian Chen ${ }^{1, *}$ \\ Li-Bo Jiang ${ }^{1, *}$ \\ Xiao-Fan Yin ${ }^{1,2}$ \\ Chong Bian' \\ Hui-Ren Wang' \\ Yi-Qun Ma' \\ Xiang-Qian $\mathrm{Li}^{\prime}$ \\ Xi-Lei Li' \\ Jian Dong' \\ 'Department of Orthopaedic \\ Surgery, Zhongshan Hospital, \\ ${ }^{2}$ Department of Orthopaedic Surgery, \\ Minhang Hospital, Fudan University, \\ Shanghai, China \\ *These authors contributed equally \\ to this work
}

\begin{abstract}
Study design: This is an experimental animal study.
Objective: The objective of this study was to compare an anterior cervical discectomy and interbody fusion of a novel polylactide/nano-sized $\beta$-tricalcium phosphate (PLA/n $\beta$-TCP) bioabsorbable self-retaining cervical fusion cage (BCFC) with an autologous bone graft and polyetheretherketone (PEEK) cages.

Background: Although PLA cervical cages have potential advantages compared with traditional materials, they are not currently routinely used in spine surgery because of undesirable effects such as the lack of osteoconductivity and osteolysis around the implant. This study involved the manufacturing of a bioabsorbable cage from PLA/n $\beta$-TCP that was then used as a device for anterior cervical discectomy and fusion (ACDF) on a goat cervical spine fusion model.
\end{abstract}

Materials and methods: Eighteen goats underwent $\mathrm{C} 3 / \mathrm{C} 4$ discectomy and were randomly divided into three groups based on the following methods: Group A ( $\mathrm{n}=6)$, an autologous bone graft; Group B ( $n=6)$, PEEK cage filled with an autologous graft; and Group C ( $n=6)$, BCFC filled with an autologous iliac bone. Radiography was performed preoperatively and postoperatively and at 1, 4, 8, and 12 weeks after the operation. Disc space height (DSH) was measured at the same time. After 12 weeks, the fused segments were harvested and evaluated with functional radiographic views, biomechanical testing, and histological analyses.

Results: Over a 12-week period, the BCFC and PEEK cage groups exhibited significantly higher DSH values than the bone graft group. Additionally, the BCFC group yielded a significantly lower range of motion in axial rotation than both the autologous bone graft and PEEK cage groups. A histologic evaluation revealed an increased intervertebral bone volume/total volume ratio and better interbody fusion in the BCFC group than in the other groups.

Conclusion: The BCFC device exhibited better results than the autologous bone graft and PEEK cages in single-level ACDF models in vivo. This device may be a potential alternative to the current PEEK cages.

Keywords: goat, cervical spine cages, bioabsorbable, PLA, $\beta$-TCP, self-retaining

\section{Introduction}

Over the last two decades, ACDF has been the standard treatment for treating degenerative conditions of the cervical spine, including degenerative disc disease, cervical spondylotic myelopathy, cervical disc prolapse, and traumatic injuries. ${ }^{1-6}$ Implantation plays an important role in neurosurgical procedures. A tricortical iliac crest bone graft has been initially applied to fill the residual space after surgical disc removal. Autologous grafts are a highly effective procedure; however, they are associated
Correspondence: Jian Dong; Xi-Lei Li Department of Orthopaedic Surgery, Zhongshan Hospital, Fudan University, I 80 Fenglin Road, Shanghai 200032, China Tel +862l 640419902907

Email dong.jian@zs-hospital.sh.cn; li.xilei@zs-hospital.sh.cn 
with significant disadvantages, including bone absorption, graft extrusion, infection, hematoma, prolonged donor site pain, and pseudoarthrosis. These deficiencies have led to a rapid increase in the use of a cervical spine interbody fusion cage. A metallic cage was first used in ACDF. The shortcomings of metallic cages have been reported, which include cage migration, subsidence, stress shielding, and obscured postoperative radiologic assessment. ${ }^{3}$ Carbon fiber and PEEK cages are radiolucent and less stiff, which allow radiologic evaluation and reduces stress shielding. . $^{2,4}$ However, carbon fiber and PEEK are still nonabsorbable materials; therefore, they cannot lead to complete biological interbody fusion. Despite satisfactory initial results, longterm problems, such as subsidence and breakage of the cage, have been noticed. The design and development of a suitable fusion cage to replace a degenerated disc are currently being performed. 5,7

A possible solution for many of these problems may be a bioabsorbable cage. PLA is currently the most popular material that is used to make bioabsorbable cages, and these cages can be designed with similar stiffness to a human bone to reduce stress shielding. In addition, the adequate postoperative assessment of interbody fusion is possible. Studies on PLA cages have been conducted to explore the effect on interbody fusion, the degradation of the cages, and the tissue response to the cages. ${ }^{8}$ However, issues regarding low primary stability with the development of cracks and some foreign body reactions with inflammatory signs have been noted, which has led to widespread skepticism about the value of bioabsorbable implants and their limited use in clinical practice. Previous studies have indicated that the incorporation of $\beta$-TCP into PLA materials can both enhance osteoconductivity and buffer acid products to reduce the inflammation around the implants. ${ }^{9}$ Additionally, nano-sized $\beta$-TCP exhibits improved mechanical properties and tunable degradability compared with micro-sized powders. Therefore, it is a potentially promising approach to design composite fusion devices that combine the advantages of the two biodegradable materials and overcome the disadvantages of PLA.

Anterior plates and screws are often employed for additional support to promote interbody fusion, reduce pseudoarthrosis and the nonunion rate, and maintain spinal curvature and intervertebral height. However, plates are associated with higher rates of adjacent-level ossification development and dysphagia and may cause complications such as plate migration, screw breakage and pullout, stress shielding, and even spinal cord and nerve injuries that require further treatment. Considering these problems, a novel bioabsorbable cervical fusion device, namely, a BCFC fabricated with the composite of PLA and nano-sized $\beta$-TCP (PLA/n $\beta$-TCP), was developed; its anchoring clips, which can implant into the endplates of vertebrae, should provide the primary biomechanical stabilization. The BCFC device showed enhanced stability in single-level ACDF models through a nondestructive biomechanical test. ${ }^{10}$ The aim of this study was to evaluate the in vivo performance of BCFC and compare it with a tricortical iliac crest bone graft and a PEEK cage in a goat cervical spine interbody fusion model. This study was designed to assess the differences in the ability to facilitate interbody fusion among three groups. The tissue response around the implantation was also observed.

\section{Materials and methods \\ Study design}

The animal study was conducted in accordance with the regulations set by the China Council on Animal Care and the protocol was revised and accepted by the ethics committee of Zhongshan Hospital, Fudan University, before beginning the experiments. Eighteen adult female Chongming white goats (2 years old, average weight $42.6 \pm 3.7 \mathrm{~kg}$ ) underwent $\mathrm{C} 3 / \mathrm{C} 4$ discectomy and fusion. The goats were randomly assigned to one of the following three groups: Group A, an autologous tricortical iliac crest bone graft $(n=6)$; Group B, a Medtronic cage (Medtronic, Minneapolis, MN, USA) filled with an autologous cancellous bone graft $(n=6)$; and Group C, BCFC filled with an autologous cancellous bone graft $(n=6)$.

The goats were prospectively evaluated for 12 weeks. Afterward, all the goats were sacrificed, and radiographic, histologic, and biomechanical evaluations were performed.

\section{Implant description}

The implants that were used for the in vivo experiment included a tricortical iliac crest bone graft (Figure 1A), a Medtronic cage that has a cylindrical, hollow center and a flat superior surface (Figure 1B), and a BCFC device that is a PLA/n $\beta$-TCP composite cage (containing $30 \mathrm{wt} \%$ $\beta$-TCP) with two anchoring clips (containing $2 \mathrm{wt} \% \beta$-TCP) (Figure 1C) that can implant into the superior and inferior endplates of vertebrae to aid in expulsion resistance with custom-made instrumentation. To allow a comparison among the different implants, bone grafts and cages of similar height, width, and depth were used (Table 1). The volume of the cages and their hollow center were determined by using the water displacement technique, according to the Archimedes principle. Additionally, the 

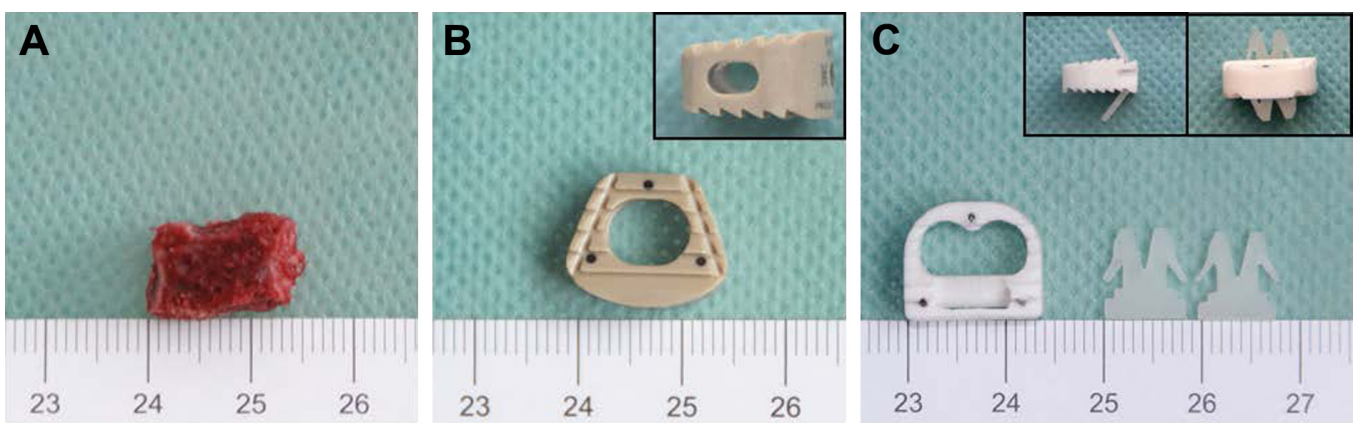

Figure I Different devices applied in the study.

Notes: (A) An autologous bone graft obtained from the anterior iliac crest. (B) A Medtronic cage having a cylindrical, hollow center and a flat superior surface. Inset: lateral view of the Medtronic cage. (C) The BCFC device, the composite of an interbody fusion cage and two anchoring clips (insets).

Abbreviation: BCFC, bioabsorbable self-retaining cervical fusion cage.

process of the PLA/n $\beta$-TCP composite can be found in the previous study. 9,10

\section{Surgical technique and postoperative care}

The animals fasted 8-12 hours before surgery and were allowed water ad libitum. In addition, the animals were administered 3.2 million units of penicillin sodium (Huabei Pharmaceutics Co. Ltd, Shijiazhuang, China) intravenously before the operation. An endotracheal tube was put to ensure an unimpeded airway. The animals underwent the operation under general anesthesia by intramuscular injection of xylazine hydrochloride (Huabei Pharmaceutics Co. Ltd). For maintenance of the anesthesia, $0.2 \%$ of chloralhydrate (Beijing Wuzhou Century Red Star Chemicals Ltd, Beijing, China) was administered intravenously, and the speed of input was adjusted according to the goats' consciousness.

The anterior part of the neck and the left iliac were prepared in a sterile manner, and a left anterolateral approach to the cervical spine was performed through a longitudinal skin incision. The longus coli muscle was elevated bilaterally with diathermy, and the position of the $\mathrm{C} 3 / \mathrm{C} 4$ level was confirmed with fluoroscopy. Distraction was achieved with a Caspar distractor (Qingniu Medical Devices Co. Ltd, Suzhou, China) followed by a total $\mathrm{C} 3 / \mathrm{C} 4$ discectomy and removal of the cartilaginous endplates with a high-speed drill to reveal bleeding bone, which resulted in an excision of $\sim 1 \mathrm{~mm}$ of each endplate. A tricortical bone graft was implanted into the intervertebral space in Group A (Figure 2A). The cages were filled with the autologous cancellous bone that was harvested from the left anterior iliac crest through a mini incision in Group B (Figure 2B and C). The BCFC cage was inserted into the disc space by using an impactor. After the implantation of the BCFC cage, two anchoring clips were placed into the upper and lower vertebrae through the anterior part of the cage to ensure the primary stabilization of the cage (Figure 2C). For wound closure, the fascia, subcutaneous tissue, and skin were approximated and closed with 1-0 Vicryl sutures (Ethicon Inc., Cornelia, GA, USA).

After extubation, the goats were transferred to a metabolic cage for observation and received 3.2 million units of penicillin sodium intramuscularly per day for 5 days. Clinical examination was performed daily for the first 7 days and then weekly. The goats were then transferred to open pastures for the duration of the study where regular observations were made.

Twelve weeks after surgery, the animals were sacrificed by pentobarbital sodium (Huabei Pharmaceutics Co. Ltd, Shijiazhuang, China) overdose. The complete cervical spine, including parts of the occiput and the first thoracic vertebrae, was then excised and cleaned from the surrounding tissue.

\section{Radiographic evaluation}

To allow for a comparable radiographic evaluation, special fixation devices for the goats' cervical spine were developed as described. Lateral and posterior-anterior digital radiographic scans were performed before and after surgery

Table I Height, width, depth, and volume of the cervical spine interbody fusion cages and bone graft

\begin{tabular}{|c|c|c|c|c|c|c|c|}
\hline Cage & Company & Materials & Height (mm) & Width (mm) & Depth (mm) & Volume $\left(\mathrm{cm}^{3}\right)$ & H-volume $\left(\mathrm{cm}^{3}\right)$ \\
\hline Bone graft & - & Bone & 7 & 14 & 12 & 1.2 & - \\
\hline Medtronic cage & Medtronic & PEEK & 7 & 14 & 13 & 1.6 & 0.5 \\
\hline BCFC & - & PLA/nano-sized $\beta$-TCP & 7 & 14 & 14 & I.I & 0.5 \\
\hline
\end{tabular}

Note: $\mathrm{H}$-volume indicates the volume of the center hollow of cages.

Abbreviations: BCFC, bioabsorbable self-retaining cervical fusion cage; $\beta$-TCP, $\beta$-tricalcium phosphate; PEEK, polyetheretherketone; PLA, polylactide. 

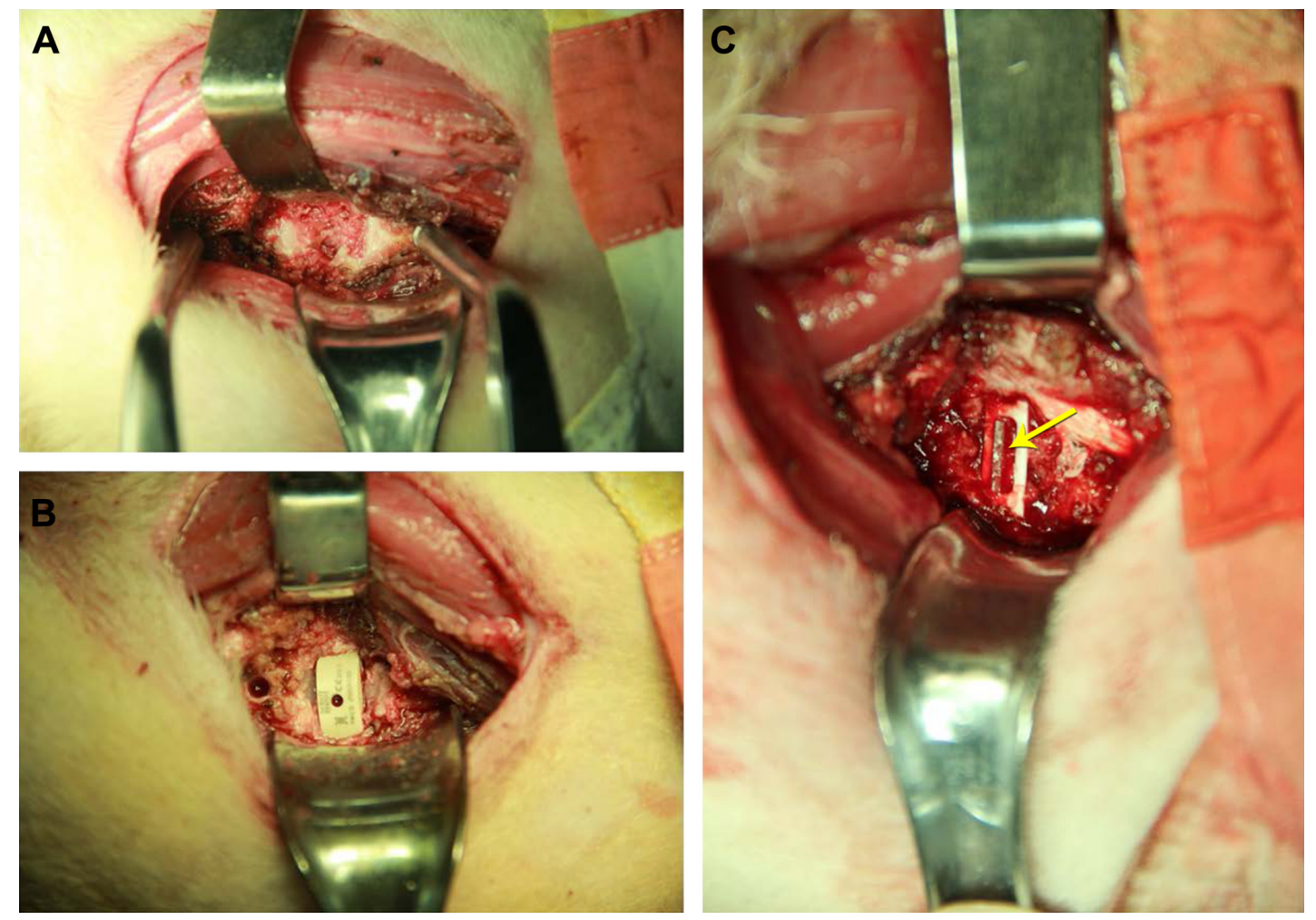

Figure 2 Different devices in the surgery.

Notes: (A) A tricortical bone graft alone. (B) A Medtronic cage filled with an autologous cancellous bone graft. (C) A BCFC filled with an autologous cancellous bone graft. The yellow arrow indicates the anchoring clip.

Abbreviation: BCFC, bioabsorbable self-retaining cervical fusion cage.

and also after 1, 4, 8, and 12 weeks. During the same time periods, the mean intervertebral disc space height (DSH) was calculated from the anterior, middle, and posterior DSH measurements ([anterior DSH + middle DSH + posterior $\mathrm{DSH}] / 3$ ) that were measured on lateral radiographs.

All the radiographs that were obtained at 12 weeks were encoded and reviewed in a blinded fashion to assess interbody fusion according to a three-point radiographic score (RS) that was described by van Dijk et al. ${ }^{11}$ The details of RS are provided in Table 2.

\section{Biomechanical analysis}

After the animals were sacrificed, the $\mathrm{C} 3 / \mathrm{C} 4$ motion segment was isolated from the cervical spine, and biomechanical testing was completed within 24 hours. The muscle tissues were cleaned without damaging the ligaments, discs, or joint capsules. The cages were not removed during the biomechanical testing. After the lower pot was rigidly fixed to the material testing apparatus, nondestructive biomechanical testing was performed by using a mechanical testing system (MTS) machine (Zwick Roell, Ulm, Germany). A pure moment was applied to the $\mathrm{C} 3$ vertebra through servomotors. Each cervical spine was subjected to an unconstrained pure moment of $\pm 1.5 \mathrm{~N} \mathrm{~m}$ at a motor rate of $1 \% \mathrm{~s}$ for a total of load/unload cycles; relaxing for 60 seconds was allowed to minimize the viscoelastic response before the data of the third cycle were used for analysis. The resultant three-dimensional range of motion (ROM) of each segment was tracked by the motional analysis system.

\section{Histological evaluation}

After radiography and biomechanical testing, the specimens were decalcified with $10 \%$ hydrochloric acid, washed under running tap water, and stored in $75 \%$ ethanol. The cages were removed from the specimens before the specimens were embedded in paraffin. The specimens were cut into

Table 2 Fusion rate after 12 weeks according to three-point RS

\begin{tabular}{llll}
\hline Cage & RS0 & RSI & RS2 \\
\hline Bone graft & $2 / 6$ & $3 / 6$ & $1 / 6$ \\
Medtronic cage & $1 / 6$ & $3 / 6$ & $2 / 6$ \\
BCFC & $0 / 6$ & $3 / 6$ & $3 / 6$ \\
\hline
\end{tabular}

Notes: RSO indicates pseudoarthrosis; RSI indicates ingrowth of bone with the cage securely fixed to vertebral bone above and below, but with a radiolucent discontinuity in the fusion mass; and RS2 indicates arthrodesis with solid bone bridging the fusion area.

Abbreviations: BCFC, bioabsorbable self-retaining cervical fusion cage; RS, radiographic score. 
$6 \mathrm{~mm}$ sections by using a Leica SP 2600 microtome (Leica Microsystems, Wetzlar, Germany). Hematoxylin-eosin, Masson's trichrome, and toluidine blue staining were performed for the transmitted light microscopy as described. Immunohistochemical staining of osteocalcin was also performed.

The histomorphologic analysis included the evaluation of bony fusion as described earlier. ${ }^{12}$ The sagittal diameter distance of $\mathrm{C} 3$ and the average preoperative DSH were determined to define the size of the region of interest (ROI) for the histomorphometrical evaluation. The complete intervertebral fusion area was included in this ROI. The following structural indexes were calculated in the ROI: bone volume/ total volume; cartilage volume/total volume; and mineralized cartilage volume/cartilage volume.

The foreign body reactions that were associated with the bioabsorbable implants were graded histologically according to the Hoffmann score. ${ }^{13}$

\section{Data and statistical analysis}

The comparison of the data was performed by using a one-way analysis of variance for the independent samples followed by Tukey's post hoc analysis of the multiple comparison procedures with a Bonferroni correction for the multiple measurements. Statistically significant differences were defined at a 95\% confidence level. The values were provided as mean \pm standard deviation. SPSS software (SPSS Inc., Chicago, IL, USA) supported the statistical evaluation.

\section{Results}

\section{Complication}

All goats recovered after surgery and were walking the evening of surgery. However, in Group B, a hematoma at the donor site of the iliac crest graft occurred on one animal, and the condition resolved without further difficulty with conservative treatment.

\section{Radiographic results}

Regarding the preoperative baseline values of all radiographic parameters, there were no intergroup differences. One week after surgery, no differences were found in DSH among the three groups. At 4, 8, and 12 weeks, DSH of both the PEEK cage and the BCFC groups was significantly greater than that of Group A $(P<0.05)$. During the complete follow-up observation, there were no significant differences in the average DSH between the PEEK cage and the BCFC groups (Figure 3).

Table 2 summarizes the results of the fusion assessments after 12 weeks of follow-up evaluation. Two specimens in
Group A, one specimen in Group B, and no specimen in Group $\mathrm{C}$ exhibited a RS of 0. One specimen in Group A, 2 specimens in Group B, and 3 specimens in Group C exhibited a RS of 2 .

\section{Biomechanical results}

The nondestructive biomechanical testing of the operative motion segments indicated no statistical significance $(P>0.05)$ in ROM among the three groups under extension loading. The results of flexion and lateral bending appeared to demonstrate a significantly higher ROM in Group A than in Groups B and C, and a more reduced ROM was observed in Group $\mathrm{C}$ than in Group B; but no significant difference was found $(P>0.05)$. However, the axial rotation $\mathrm{ROM}$ indicated a significantly reduced $\mathrm{ROM}$ in the BCFC group compared with the PEEK cage group $(P<0.05)$ (Figure 4).

\section{Histologic results}

The results of histomorphometrical analysis are depicted in Table 3. The histomorphological and histomorphometrical analyses revealed a significantly progressed intervertebral bone matrix formation, an increased intervertebral bone volume/total volume ratio, and an advanced interbody fusion in the BCFC cage group compared with the other groups.

BCFC demonstrated minimal evidence of foreign body reactions and osteolysis in six animals (Figure 5), and no spontaneous cracks occurred in six BCFC cages.

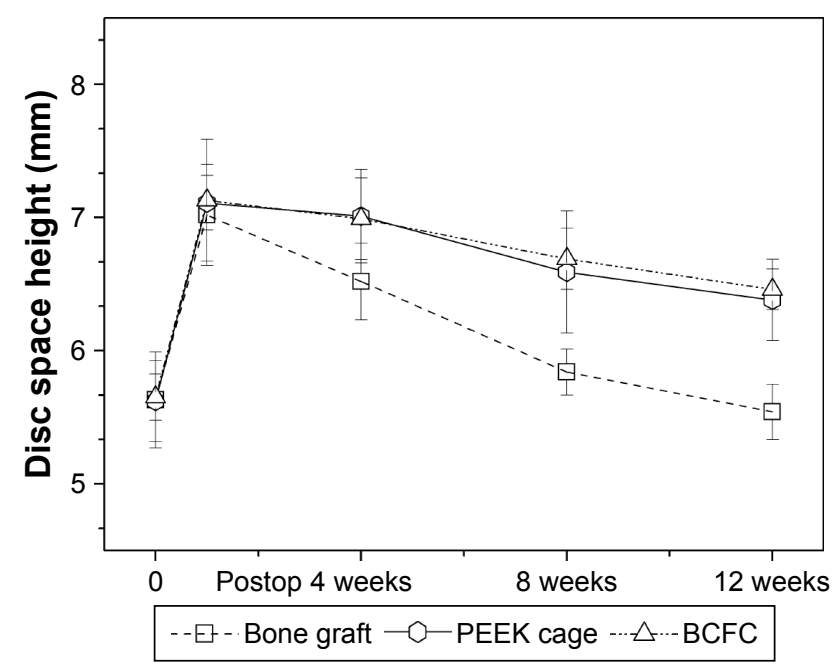

Figure 3 Radiographic analysis: average DSH of the different groups throughout the observation period.

Abbreviations: DSH, disc space height; PEEK, polyetheretherketone; BCFC, bioabsorbable self-retaining cervical fusion cage. 


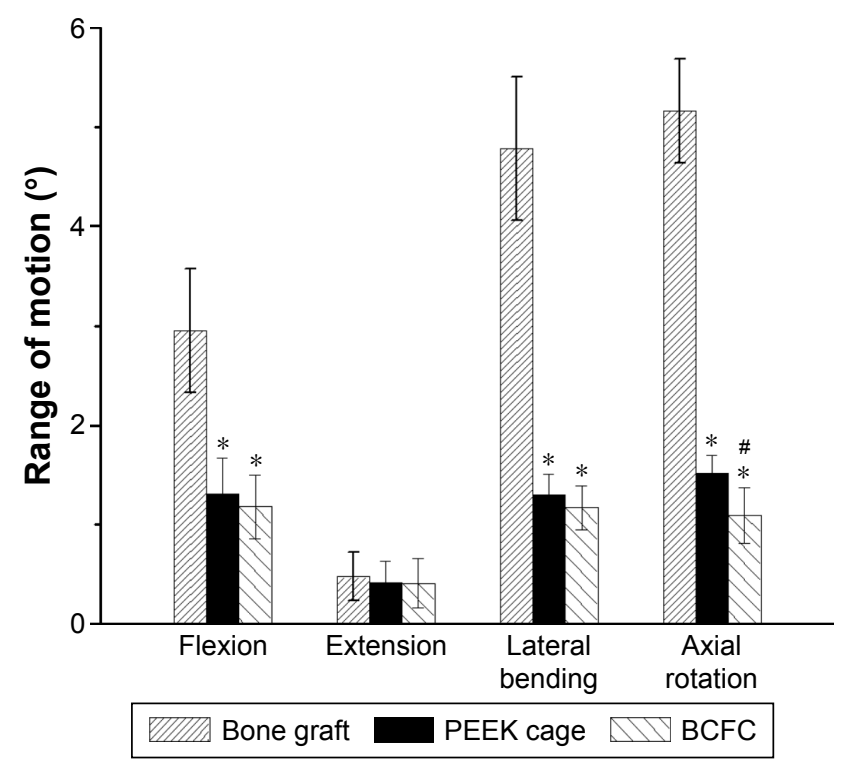

Figure 4 Graph showing the variation in biomechanical ROM in all loading models for all groups.

Note: * and \# indicate significance with respect to bone graft and Medtronic cage, respectively $(P<0.05)$.

Abbreviations: ROM, range of motion; PEEK, polyetheretherketone; BCFC, bioabsorbable self-retaining cervical fusion cage.

\section{Discussion}

Most of the studies on bioabsorbable cages focus on PLA and its copolymer, ${ }^{1-17}$ which have $\sim 30$ years of clinical application, including for sutures, plates, and screws in different orthopedic surgeries. ${ }^{18,19}$ Although cervical fusion cages that are made of PLA generally work very well,, 15,17,20,21 the acidic degradation products of PLA can cause asepsis inflammation and osteolysis, ${ }^{22-24}$ which will deteriorate the microenvironment of bone healing. In addition, PLA cannot offer the capacity to osteoconduct.

$\beta$-TCP has been widely used in surgical procedures because of its degradation behavior and similar calcium/ phosphorus $(\mathrm{Ca} / \mathrm{P})$ ratio to hydroxyapatite, ${ }^{25,26}$ which is considered the modified form of up to $50 \%$ by volume and $70 \%$ by weight of human bone. ${ }^{27}$ However, significant disadvantages have been observed. It is difficult to process $\beta$-TCP into an ideal shape or porous structure because of its brittleness, and the use of $\beta$-TCP alone is obviously limited. It has also been employed as a filler to improve osteoconductivity and to offset the acid degradation products of PLA. ${ }^{21,28-30}$ Thus, it may perform well in restraining the inflammatory response that is caused by the high acidity of the PLA monomer.

Based on this information, it is wise to combine these materials to form a composite of PLA/ $\beta$-TCP with a particular approach. According to the previous study, ${ }^{30}$ the cage and anchoring clips were fabricated with different contents of nano-sized $\beta$-TCP according to the data. The $\mathrm{PLA} /$ nano-sized composite rods were obtained from an axial compression molding process and were investigated to screen the optimal components' proportion for fabricating the cage and anchoring clips from different viewpoints such as degradation, mechanical properties, and the biocompatibility of the composites. Finally, it was concluded that the PLA/30 n $\beta$-TCP scaffolds exhibited similar osteogenesis compared with the other groups and a better performance of mechanical properties.

The anterior cervical plating enhances the stability of the cervical spine and may avoid the risk of pseudoarthrosis. However, the plate-related complications are a cause of significant concern. The rate of plate-related complications increases in direct proportion to the number of fused segments. Thus, the key of surgery is to address the cervical plate and maintain its stability. As far as the authors are concerned, one of the most successful cervical surgeries is ACDF, which relies on a thorough decompression and achievement of biomechanical stability. The previous in vitro study revealed that a BCFC provides better initial biomechanical stability than two types of PEEK traditional cages. ${ }^{8}$ The novel BCFC may be a promising alternative to the current PEEK cages and anterior plate devices.

Long-term clinical studies ${ }^{31-34}$ have demonstrated an increased incidence of pathologies in the motion segments that are adjacent to the fused segment. Many scholars have emphasized the altered biomechanics at the adjacent segment after fusion that lead to increased mobility, ${ }^{31-33}$ loading, intradiscalpressure, ${ }^{35,36}$ and accelerated disc degeneration..$^{37,38}$

Table 3 SDD (baseline), BV/TV, CV/TV, and mCV/CV calculations in the ROI after histomorphometrical analysis

\begin{tabular}{llll}
\hline Index & Bone graft & Medtronic cage & BCFC \\
\hline SDD $(\mathrm{mm})$ & $17.1 \pm 0.6(16.2-17.9)$ & $17.3 \pm 0.8(15.9-18.1)$ & $17.3 \pm 0.6(16.3-17.9)$ \\
BV/TV $(\%)$ & $23.4 \pm 2.2(18.2-27.3)$ & $27.1 \pm 2.6(21.1-30.0)^{*}$ & $30.3 \pm 2.4(26.2-35.3)^{* . \#}$ \\
CV/TV $(\%)$ & $12.6 \pm 2.0(8.3-14.9)$ & $5.6 \pm 1.5(4.2-7.7)^{*}$ & $5.2 \pm 0.9(4.1-6.2)^{*}$ \\
$\mathrm{mCV} / \mathrm{CV}(\%)$ & $5.9 \pm 0.8(4.0-7.3)$ & $5.3 \pm 0.6(4.2-6.4)$ & $5.4 \pm 0.4(4.6-5.8)$ \\
\hline
\end{tabular}

Notes: $* P<0.05$ in comparison with the bone graft. ${ }^{*}<0.05$ in comparison with the bone graft and the Medtronic cage. Data presented as mean \pm standard deviation. Abbreviations: BCFC, bioabsorbable self-retaining cervical fusion cage; BV/TV, bone volume/total volume; CV/TV, cartilage volume/total volume; mCV/CV, mineralized cartilage volume/cartilage volume; ROI, region of interest; SDD, sagittal diameter distance. 


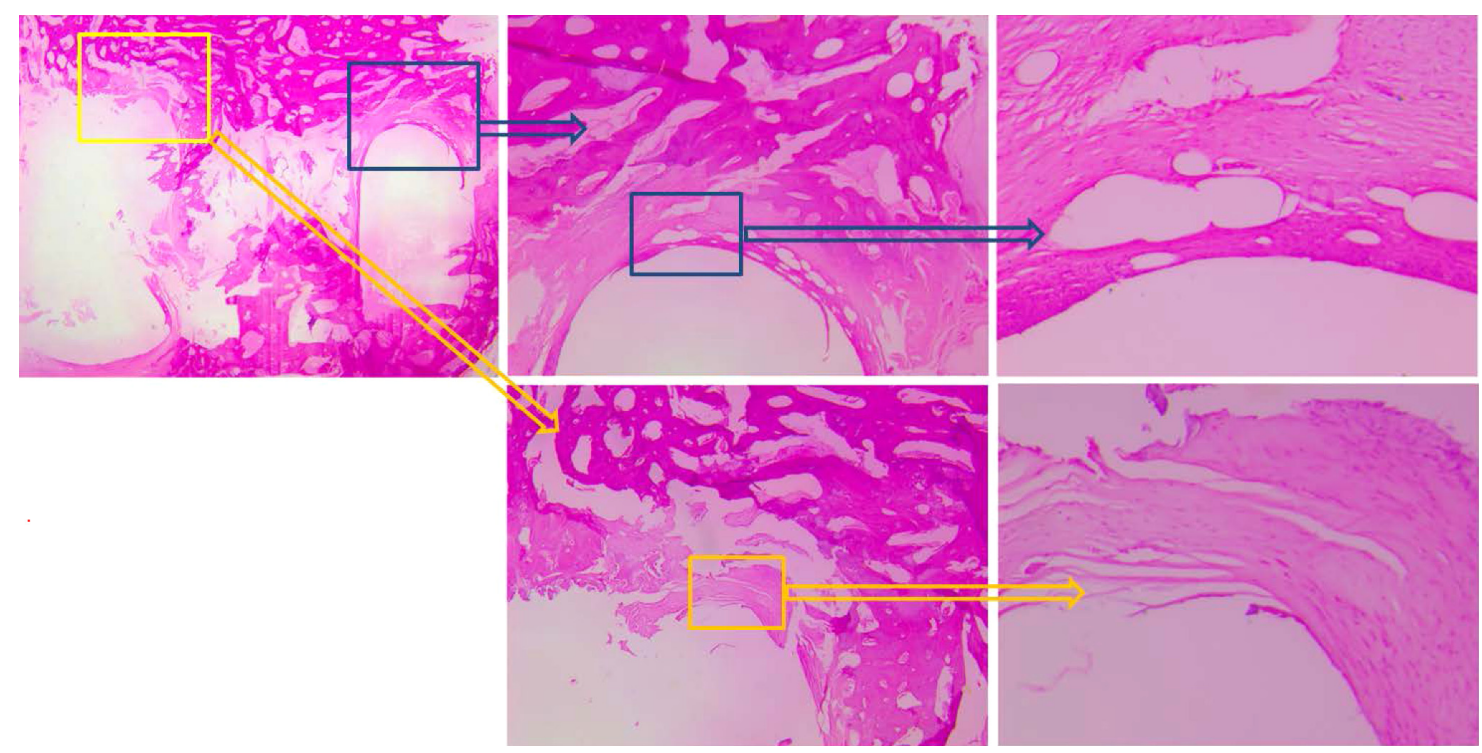

Figure 5 Foreign body reactions of the bioabsorbable implants were graded histologically according to the Hoffmann score. The BCFC cage demonstrated hardly any foreign body reaction. Depicted is a grade 0 foreign body reaction.

Abbreviation: BCFC, bioabsorbable self-retaining cervical fusion cage.

Nagata et $\mathrm{al}^{39}$ reported that the motion segments adjacent to the fused segment exhibit an increased ROM, and the increase in motion at the adjacent segments was proportional to the number of fused vertebrae. Gore and Sepic ${ }^{40}$ noted that, regardless of the fusion approach, cervical fusion increased the mobility of the adjacent segments and facilitated degenerative change. According to a follow-up of $>7$ years, $31.3 \%$ of patients suffered a recurrence of pain and $17.8 \%$ of patients required surgery to relieve it.

In contrast to these findings, other studies have suggested that natural progression at adjacent levels after fusion plays a key role in adjacent spinal diseases. ${ }^{31-40}$ Kellgren and Lawrence $^{41}$ reported that physiologic aging of the cervical spine was observed by plain radiographs in healthy volunteers who were $>50$ years of age.

Another more important finding is that Gore et $\mathrm{al}^{42}$ reviewed the radiographic changes in people who had undergone cervical fusion and in a population without neck problems that had not undergone neck surgery at a mean follow-up of 5 years. From their results, the incidence of adjacent-segment pathology showed no difference between the patients who underwent surgery and their asymptomatic counterparts.

Over the course of a clinical study with $>10$ years of follow-up, Chung et $\mathrm{al}^{43}$ confirmed that two factors, namely, changed biomechanical stresses and the natural history of spine disc degeneration, resulted in the adjacent-segment pathology.

In this study, it was demonstrated that the BCFC group had a significantly lower ROM in axial rotation compared with both the autologous tricortical iliac crest bone graft and PEEK cage groups, which manifested a better stability from a biomechanical viewpoint, and thus BCFC could be regarded as a better device to hinder adjacent segment degeneration (ASD). This alternative may be particularly important in cases where sufficient methods are not available to intervene in the natural progression of ASD.

\section{Limitations}

Unavoidably, there were some limitations to this study. First, the use of goat spines in vivo causes an inevitable bias. Differences undoubtedly exist between the biomechanical performance of implants in goats and the biomechanical performance of implants in humans. Second, the sample size was small and therefore inadequate. Thus, the results must be validated by further studies with a larger sample size. Finally, this study focused on ROM in the operation segment. The facet joints, which are a crucial component of the "triple joint complex," should be evaluated in future research.

This study confirmed that the BCFC device played a positive role in a goat model after ACDF. Compared with an autologous tricortical iliac crest bone graft and PEEK cage groups, the BCFC device group exhibited better results in biomechanical stability, intervertebral bone volume/total volume, and interbody fusion. Considering all the cage fusions in the single-level ACDF in the goat model in vivo, the novel BCFC device may be a promising alternative to the current PEEK cages. However, some limitations of this in vivo study of goat model should be addressed in the future. 


\section{Conclusion}

A PLA/n $\beta$-TCP BCFC was assessed in a goat model. At 12 weeks after implantation surgery, the BCFC device yielded better results than an autologous bone graft and PEEK cages in single-level ACDF models, as assessed by nondestructive biomechanical testing. This BCFC device may be a potential alternative to the current PEEK cages and further investigation is warranted.

\section{Acknowledgments}

This work was supported by funds from the State Key Project of Research and Development (2016YFC1100300), National High-tech R\&D Program (863 Program) (2015AA033703), and Natural Science Foundation of China (81572629, 81372002, and 81401820). The devices and drugs were Food and Drug Administration-approved or approved by the corresponding national agency for this study. There were no relevant financial activities outside the submitted work.

\section{Disclosure}

The authors report no conflicts of interest in this work.

\section{References}

1. Daentzer D, Willbold E, Kalla K, et al. Bioabsorbable interbody magnesium-polymer cage: degradation kinetics, biomechanical stiffness, and histological findings from an ovine cervical spine fusion model. Spine (Phila Pa 1976). 2014;39:E1220-E1227.

2. Hermansen A, Hedlund R, Vavruch L, Peolsson A. A comparison between the carbon fiber cage and the cloward procedure in cervical spine surgery: a ten- to thirteen-year follow-up of a prospective randomized study. Spine (Phila Pa 1976). 2011;36:919-925.

3. Kaden B, Swamy S, Schmitz HJ, Reddemann H, Fuhrmann G, Gross U. Titanium implant as an alternative possibility in fusion of the cervical vertebrae - initial clinical experiences. Zentralbl Neurochir. 1993;54:166-170.

4. Liao JC, Niu CC, Chen WJ, Chen LH. Polyetheretherketone (PEEK) cage filled with cancellous allograft in anterior cervical discectomy and fusion. Int Orthop. 2008;32:643-648.

5. Marotta N, Landi A, Tarantino R, Mancarella C, Ruggeri A, Delfini R. Five-year outcome of stand-alone fusion using carbon cages in cervical disc arthrosis. Eur Spine J. 2011;20 (Suppl 1):S8-S12.

6. Nayak AN, Stein MI, James CR, et al. Biomechanical analysis of an interbody cage with three integrated cancellous lag screws in a twolevel cervical spine fusion construct: an in vitro study. Spine J. 2014;14: 3002-3010.

7. Yang JJ, Yu CH, Chang BS, Yeom JS, Lee JH, Lee CK. Subsidence and nonunion after anterior cervical interbody fusion using a standalone polyetheretherketone (PEEK) cage. Clin Orthop Surg. 2011;3: $16-23$.

8. Frost A, Bagouri E, Brown M, et al. Osteolysis following resorbable poly-L-lactide-co-D, L-lactide PLIF cage use: a review of cases. Eur Spine J. 2012;21(3):449-454.

9. Cao L, Duan PG, Wang HR, et al. Degradation and osteogenic potential of a novel poly(lactic acid)/nano-sized $\beta$-tricalcium phosphate scaffold. Int J Nanomedicine. 2012;7:5881-5888.
10. Cao L, Duan PG, Li XL, et al. Biomechanical stability of a bioabsorbable self-retaining polylactic acid/nano-sized $\beta$-tricalcium phosphate cervical spine interbody fusion device in single-level anterior cervical discectomy and fusion sheep models. Int J Nanomedicine. 2012;7: 5875-5880.

11. van Dijk M, Smit TH, Burger EH, Wuisman PI. Bioabsorbable polyL-lactic acid cages for lumbar interbody fusion: three-year follow-up radiographic, histologic, and histomorphometric analysis in goats. Spine (Phila Pa 1976). 2002;27:2706-2714.

12. Kandziora F, Pflugmacher R, Scholz M, Eindorf T, Schnake KJ, Haas NP. Bioabsorbable interbody cages in a sheep cervical spine fusion model. Spine (Phila Pa 1976). 2004;29:1845-1855.

13. Hoffmann R, Weller A, Helling HJ, Krettek C, Rehm KE. Local foreign body reactions to biodegradable implants. A classification. Unfallchirurg. 1997;100:658-666.

14. Coe JD, Vaccaro AR. Instrumented transforaminal lumbar interbody fusion with bioresorbable polymer implants and iliac crest autograft. Spine (Phila Pa 1976). 2005;30:S76-S83.

15. Wuisman PIJM, Smit TH. Bioresorbable polymers: heading for a new generation of spinal cages. Eur Spine J. 2006;15:133-148.

16. Thomas KA, Toth JM, Crawford NR, et al. Bioresorbable polylactide interbody implants in an ovine anterior cervical discectomy and fusion model: three-year results. Spine (Phila Pa 1976). 2008;33: 734-742.

17. Jiya T, Smit T, Deddens J, Mullender M. Posterior lumbar interbody fusion using nonresorbable poly-ether-ether-ketone versus resorbable poly-L-lactide-co-D,L-lactide fusion devices: a prospective, randomized study to assess fusion and clinical outcome. Spine (Phila Pa 1976). 2009;34:233-237.

18. Deguchi M, Cheng BC, Sato K, Matsuyama Y, Zdeblick TA. Biomechanical evaluation of translaminar facet joint fixation. A comparative study of poly-L-lactide pins, screws, and pedicle fixation. Spine (Phila Pa 1976). 1998;23(12):1307-1312; discussion 1313.

19. Bostman OM. Osteoarthritis of the ankle after foreign-body reaction to absorbable pins and screws: a three- to nine-year follow-up study. J Bone Joint Surg Br. 1998;80(2):333-338.

20. Soderlund CH, Pointillart V, Pedram M, Andrault G, Vital JM. Radiolucent cage for cervical vertebral reconstruction: a prospective study of 17 cases with 2-year minimum follow-up. Eur Spine J. 2004;13:685-690.

21. Debusscher F, Aunoble S, Alsawad Y, Clement D, Le Huec JC. Anterior cervical fusion with a bio-resorbable composite cage (beta TCP-PLLA): clinical and radiological results from a prospective study on 20 patients. Eur Spine J. 2009;18:1314-1320.

22. Bostman OM. Absorbable implants for the fixation of fractures. J Bone Joint Surg Am. 1991;73:148-153.

23. Bucholz RW, Henry S, Henley MB. Fixation with bioabsorbable screws for the treatment of fractures of the ankle. J Bone Joint Surg Am. 1994;76:319-324.

24. Suganuma J, Alexander H. Biological response of intramedullary bone to poly-L-lactic acid. J Appl Biomater. 1993;4:13-27.

25. Wang J, Qu L, Meng X, Gao J, Li H, Wen G. Preparation and biological properties of PLLA/beta-TCP composites reinforced by chitosan fibers. Biomed Mater. 2008;3(2):025004.

26. Crouzier T, Sailhan F, Becquart P, Guillot R, Logeart-Avramoglou D, Picart C. The performance of BMP-2 loaded TCP/HAP porous ceramics with a polyelectrolyte multilayer film coating. Biomaterials. 2011; 32(30):7543-7554.

27. Junqueira LC, Carneiro J. Basic Histology: Text \& Atlas. 10th ed. New York: McGraw-Hill; 2003:144. ISBN 0-07-137829-4.

28. Barber FA, Boothby MH. Bilok interference screws for anterior cruciate ligament reconstruction: clinical and radiographic outcomes. Arthroscopy. 2007;23(5):476-481.

29. Barber FA, Dockery WD. Long-term absorption of beta-tricalcium phosphate poly-L-lactic acid interference screws. Arthroscopy. 2008; 24(4):441-447. 
30. Huang J, Zhang L, Chu B, Peng X, Tang S. Repair of bone defect in caprine tibia using a laminated scaffold with bone marrow stromal cells loaded poly (L-lactic acid)/ $\beta$-tricalcium phosphate. Artif Organs. 2011;35(1):49-57.

31. Baba H, Furusawa N, Imura S, Kawahara N, Tsuchiya H, Tomita K. Late radiographic findings after anterior cervical fusion for spondylotic myeloradiculopathy. Spine (Phila Pa 1976). 1993;18:2167-2173.

32. Dohler JR, Kahn MR, Hughes SP. Instability of the cervical spine after anterior interbody fusion. A study on its incidence and clinical significance in 21 patients. Arch Orthop Trauma Surg. 1985;104:247-250.

33. Woesner ME, Mitts MG. The evaluation of cervical spine motion below C2: a comparison of cineroentgenographic and conventional roentgenographic methods. Am J Roentgenol Radium Ther Nucl Med. 1972;115:148-154.

34. Clements DH, O'Leary PF. Anterior cervical discectomy and fusion. Spine. 1990;15:1023-1025.

35. Matsunaga S, Kabayama S, Yamamoto T, Yone K, Sakou T, Nakanishi K. Strain on intervertebral discs after anterior cervical decompression and fusion. Spine (Phila Pa 1976). 1999;24:670-675.

36. Pospiech J, Stolke D, Wilke HJ, Claes LE. Intradiscal pressure recordings in the cervical spine. Neurosurgery. 1999;44:379-384.
37. Schwab JS, Diangelo DJ, Foley KT. Motion compensation associated with single-level cervical fusion: where does the lost motion go? Spine (Phila Pa 1976). 2006;31:2439-2448.

38. Ragab AA, Escarcega AJ, Zdeblick TA. A quantitative analysis of strain at adjacent segments after segmental immobilization of the cervical spine. J Spinal Disord Tech. 2006;19:407-410.

39. Nagata H, Schendel MJ, Transfeldt EE, Lewis JL. The effects of immobilization of long segments of the spine on the adjacent and distal facet force and lumbosacral motion. Spine (Phila Pa 1976). 1993; 18:2471-2479.

40. Gore DR, Sepic SB. Anterior discectomy and fusion for painful cervical disc disease. A report of 50 patients with an average follow-up of 21 years. Spine (Phila Pa 1976). 1998;23:2047-2051.

41. Kellgren JH, Lawrence JS. Osteo-arthrosis and disk degeneration in an urban population. Ann Rheum Dis. 1958;17:388-397.

42. Gore DR, Gardner GM, Sepic SB, Murray MP. Roentgenographic findings following anterior cervical fusion. Skeletal Radiol. 1986;15: 556-559.

43. Chung JY, Kim SK, Jung ST, Lee KB. Clinical adjacent-segment pathology after anterior cervical discectomy and fusion: results after a minimum of 10-year follow-up. Spine J. 2014;14(10):2290-2298.
International Journal of Nanomedicine

\section{Publish your work in this journal}

The International Journal of Nanomedicine is an international, peerreviewed journal focusing on the application of nanotechnology in diagnostics, therapeutics, and drug delivery systems throughout the biomedical field. This journal is indexed on PubMed Central, MedLine, CAS, SciSearch $®$, Current Contents $\AA /$ Clinical Medicine,

\section{Dovepress}

Journal Citation Reports/Science Edition, EMBase, Scopus and the Elsevier Bibliographic databases. The manuscript management system is completely online and includes a very quick and fair peer-review system, which is all easy to use. Visit http://www.dovepress.com/ testimonials.php to read real quotes from published authors. 